\title{
Transthyretin Antisense Oligonucleotide ISIS-TTR Rx
}

National Cancer Institute

\section{Source}

National Cancer Institute. Transthyretin Antisense Oligonucleotide ISIS-TTR RX. NCI

Thesaurus. Code C121667.

An antisense oligonucleotide (ASO) targeting transthyretin (TTR), which has potential use in the treatment of TTR amyloidosis (ATTR). Upon subcutaneous administration, TTR ASO ISIS-TTR Rx targets and binds to messenger RNA (mRNA) for both variant and wildtype forms of TTR inside liver cells, thereby inhibiting translation of both mutant and wildtype TTR. Inhibition of TTR protein synthesis lowers TTR blood levels and decreases the amount of and/or prevents TTR amyloid deposits, which accumulate in and cause damage to various body org ans and tissues. ATTR is caused by mutations in the TTR gene, which lead to TTR protein misfolding; misfolded wild-type and mutant forms of TTR protein accumulate in tissues as amyloid deposits in most ATTR patients. 